\title{
Focus on
}

\section{Physical Principles of Protein Behavior in the Cell}

$\mathbf{W}$ living cell? To address this question, it is of central importance to understand the organization of the proteome, as proteins are involved in essentially all the processes that take place in the cell, including those required for the control of gene expression and metabolism. We are witnessing an explosion of novel experimental high-throughput techniques and theoretical analysis methods to elucidate the mechanisms of protein regulation, localization, and interaction. The physical principles underlying these advances are the subject of this Focus Issue of PROTEOMICS. The Focus Issue has been developed from an interdisciplinary conference that took place at the Max Planck Institute for the Physics of Complex Systems in Dresden, Germany, October 26-30, 2009. The event brought together researchers from all major areas relevant to understanding protein behavior in the cell, including protein expression, localization, function, folding, misfolding, and aggregation. The present Focus Issue brings together a selection of papers where opinion leaders in the field discuss the manner in which existing concepts and methods have made it possible to carry out exciting new studies of the networks of interacting molecules that underlie the myriad biological pathways through which cells maintain homeostasis and promote development. There is increasing expectation that in-depth research addressing the physical principles that underlie protein behavior in the cell will greatly improve existing methods for characterizing and predicting structures, interactions, and functions of proteins, for modeling their evolution, and ultimately for suggesting new rational approaches for treating human diseases.

The topics covered in this Focus Issue are quite broad, ranging from experimental studies to computer modeling. Müller and co-workers investigate the energy landscape in the unfolding reaction of the E. coli outer membrane protein $\mathrm{G}$ using dynamic single-molecule force spectroscopy and relate it to functional parameters of the protein such as rigidity and flexibility. Wilke and collaborators study the association between optimal codon usage and protein structural properties in the genomes of four model organisms and show that aggregation propensity and solvent accessibility have independent effects on codon usage. The relationship between stability and folding properties of a protein sequence in vitro and in situ with its aggregation properties in vivo is studied by Ventura and co-workers using SH3 domains as example. This paper is followed by one by Schroeder and collaborators describing the structural modeling of the histone methyltransferase complex Set1C in yeast, which consists of eight subunits, through homology modeling and computational docking. Then, Schwille and her collaborators investigate the relative abundance of bitopic membrane proteins and the amino acid composition of their transmembrane domains in different taxa and determine how the context of the transmembrane domains of bitopic receptors function in the assembly of dimers, whereas Marcotte and his group study the conservation of mRNA and protein abundances comparing seven different species, showing that protein abundances are generally more conserved than mRNA abundances. The Focus Issue is concluded by a paper by Lilley and collaborators who describe a refined bioinformatic approach to assign proteins of uncharacterized localization to subcellular structures.

The Editors wish to thank the Editor-in-Chief of PROTEOMICS, Professor Mike Dunn, the Managing Editor, Dr. Hans Joachim Kraus, and the publisher Wiley-VCH for offering

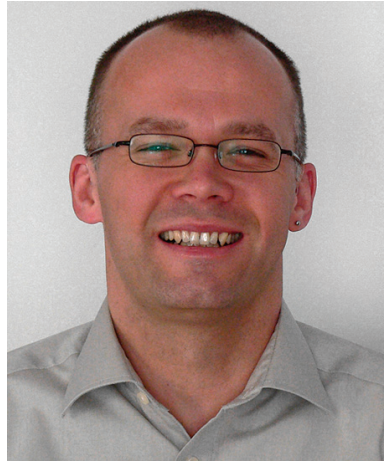

Markus Porto

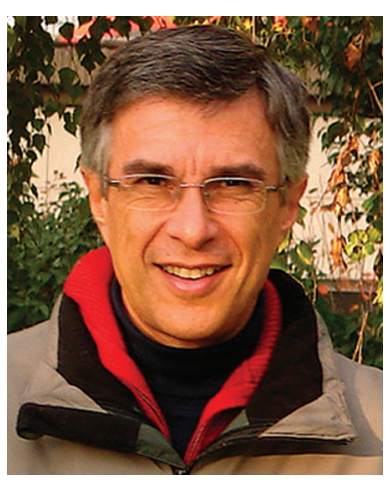

H. Eduardo Roman

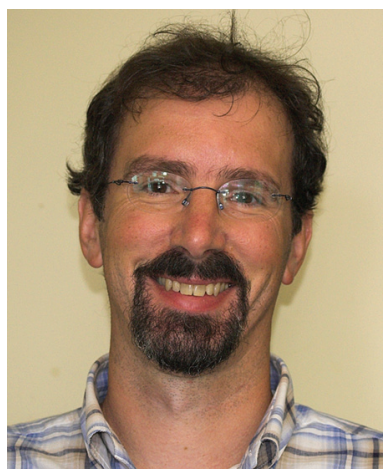

Michele Vendruscolo 
them the opportunity to prepare this Focus Issue. They wish to warmly thank all the authors for exciting contributions for the issue, and the reviewers for their critical and highly qualified evaluation of the contributions. The Editors would also like to thank the Max Planck Institute for the Physics of Complex Systems for financing and hosting the interdisciplinary conference which formed the starting point for the Focus Issue. In particular they would like to thank Dr. Sergej Flach (head of the conference program) for his very helpful support and Mrs. Claudia Pönisch (conference secretary) for her very efficient organization.

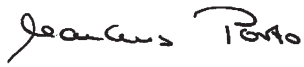

Markus Porto

Cologne
Eduardo Romain.

H. Eduardo Roman Dresden

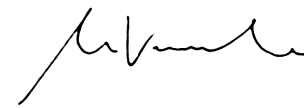

Michele Vendruscolo Cambridge 